\title{
HIGH-FIDELITY OPTIMIZATION FRAMEWORK FOR HELICOPTER ROTORS
}

\author{
Manfred Imiela, German Aerospace Center (DLR), Institute of Aerodynamics and Flow \\ Technology, Lilienthalplatz 7, 38108 Braunschweig, Germany, Manfred.Imiela@dlr.de
}

\begin{abstract}
An optimization framework for helicopter rotors based on high-fidelity coupled CFD/CSM analyses is presented. For this purpose the optimization software DAKOTA has been linked to a parametric geometry unit, a mesh generation unit and a fluid-structure module which consists of the DLR flow solver FLOWer coupled with the Comprehensive Rotorcraft Code HOST from Eurocopter.

The optimization framework is first applied to various optimization problems in hover starting with the easy task of optimizing the twist rate for the 7A model rotor. The complexity of the optimizations is increased step by step and finishes with an optimization case involving all seven design parameters showing its superiority over simpler optimization problems with respect to the achieved improvement.

In the next step the framework is operated in forward flight condition also for the optimization of the twist rate. Small improvements with respect to the $7 \mathrm{~A}$ rotor are made though indicating the conflicting nature of hover and forward flight requirements. Thus a multi-objective optimization for the twist of the $7 \mathrm{~A}$ rotor is carried out.
\end{abstract}

\begin{tabular}{ll}
$c$ & \multicolumn{1}{c}{ Notation } \\
$c_{e}$ & Ehord \\
$r, R$ & Radius \\
$\mathrm{C}_{\mathrm{q}}$ & Torque Coefficient \\
$\mu$ & Forward Flight Ratio \\
$\theta_{0}$ & Collective Pitch Angle \\
$\vec{F}$ & Vector of aerodynamic loads \\
$\vec{F}_{2 D}$ & Vector of aerod. loads from HOST \\
$\mathrm{F}_{\mathrm{Obj}}$ & Objective Function \\
$\mathrm{FM}$ & Figure of Merit \\
$\mathrm{FF}$ & Forward Flight \\
EGO & Efficient Global Optimization \\
CONGRA & Conjugate Gradient \\
DACE & Design and Analysis of Computer \\
EIF & Experiments \\
WOF & Expected Improvement Function \\
\end{tabular}

\section{INTRODUCTION}

The design of helicopter rotor blades is a quite challenging task. While high fidelity computer analyses in the fixed wing community are widely employed today, the rotary wing community still relies heavily on low fidelity models. Although being less time consuming, the ability of these models to reproduce the behaviour of the physical model vanishes quickly with increasing complexity of the geometry. Since CFD has reached a sophisticated level of maturity, manufacturers want to integrate these methods into their design process. Because of the high aspect ratio of rotor blades fluid-structure-interaction needs to be taken into account. This also helps reducing the number of design cycles.

Most studies during the last 30 years such as [5] and [19] were devoted to aeroelastic and dynamic optimization with the aim of reducing vibratory loads and dynamic stresses. The majority of these works has relied on simple aerodynamic models based on blade element momentum theory because the application of CFD inside the optimization was prohibitively expensive. In recent years some works such as [6], [7], [16], [17], [18] have put their focus on the optimization of aerodynamic efficiency. While these studies have already incorporated CFD analysis tools within the optimization loop either the amount of computations or the number of design scenarios was limited. Therefore the new rotor blade was only optimal for a single flight condition or the uncertainty with respect to the efficiency improvements was high. This is due to the known deficiency of blade element theory to accurately predict complex 3D flow phenomena which prevail especially in the tip region of the blade.

The goal of this paper is to describe an optimization approach for helicopter rotor blades based on a weak fluid-structure coupling scheme combined with an optimization algorithm in order to improve the performance of the rotor in hover and forward flight. The CFD analysis on the one side is realized with the 3D Navier-Stokes solver FLOWer. In hover steady computations are used, while in forward flight unsteady time-accurate evaluations for one blade are carried out until a periodic solution is reached. On the other side blade dynamics and elasticity are taken into account by the Comprehensive Rotorcraft Code HOST from Eurocopter. The structural model 
consists of an extended 1D Euler-Bernoulli beam model. The optimization procedure is focused on the aerodynamic performance. Periodic loads and geometric changes of the blade planform are communicated to the structural model. The structural model itself though is not modified during the optimization. In order to assess the effectivity of different optimization algorithms a comparison between three different types of algorithms has been done.

First the general strategy of the optimization procedure is introduced and the functionality of the different optimization algorithms is explained. Secondly the parameterization, the grid generation, the flow and structural simulation tools as well as the weak coupling procedure are described. Subsequently optimization results for various design variables and flight scenarios are presented. While optimizations in hover include up to seven design variables, forward flight and multi-objective optimizations contain only one design parameter.

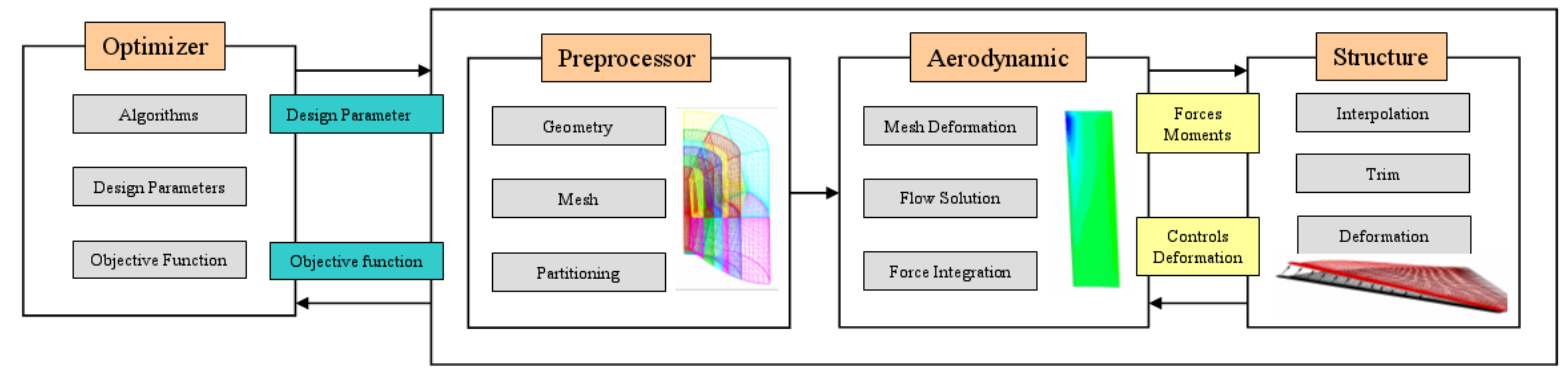

Figure 1: Flowchart of the optimization framework

\section{OPTIMIZATION FRAMEWORK}

The optimization framework as shown in Figure 1 consists of three elements, i.e. the optimizer, the preprocessing module and the fluid-structure module. The DAKOTA-Software from Sandia Labs [1] is used as optimization tool. It contains different optimization algorithms and steers the overall process by generating the design parameter sets, starting the individual evaluations and collecting the result from each analysis. The parameter set is then passed to the preprocessing unit where the mesh is created. The preprocessor starts with a series of 2D profiles which are lined up on the quarter chord line along the blade radius. The resulting $3 \mathrm{D}$ blade surface is then transferred to the grid generator where the volume mesh of the computational domain is generated. In a last step the monoblock grid is partitioned into multiple blocks in order to make it applicable to a parallel computation.

The fluid-structure module is initiated by a trim computation with HOST. This delivers the dynamic response of the rotor and the elastic deformation which serve as input for the flow computation. After the periodic coupling has been carried out for a predefined number of iterations, the aerodynamic coefficients are extracted and passed to the optimizer which decides upon the next set of design parameters. The process is continued until the improvement falls below a predefined threshold.

\subsection{Optimization algorithms}

Optimization algorithms can be categorized into three different types: deterministic methods, probabilistic methods and surrogate methods. Most algorithms of the first group rely on a relatively simple mathematical background. They are easy to implement and behave well on non-noisy, unimodal functions. While probabilistic methods such as genetic algorithms usually have a higher potential in finding the global optimum, they are considered obsolete for this study because of the numerous evaluations which are necessary for the convergence in combination with the lengthy CFD analyses. Algorithms of the third group, namely the surrogate methods, pose a good compromise between the capability of finding the global optimum and the effort needed to do so. Three different algorithms have been chosen for comparison, e.g. a gradient-based method, termed CONGRA, a nongradient-based method, called Subplex and a surrogate method, named EGO.

\subsubsection{CONGRA}

The well-known conjugate gradient method uses function values as well as gradient information in order to determine a new search direction. Since analytic gradient information is not available it must be deduced from finite differencing. Although this operation can be done in parallel, it can become costly if many design variables are involved. The subsequent line search is conducted until no further improvement is observed. At this point a new search direction has to be computed. This can be done e.g. according to the formula of Fletcher-Reeves which takes into account the search direction from former iterations. The conjugate gradient method is superior to the simpler steepest descent method on objective functions where the curvature highly differs in different dimensions. Theoretically the method converges in $\mathrm{n}$ steps, with $\mathrm{n}$ being the number of design variables. Though this is only true for smooth convex functions. 


\subsubsection{Subplex}

The Subplex method belongs to the group of gradient-free algorithms also called pattern search algorithms. Its main advantage is its robustness and the fact that the objective function does not need to be differentiable. The necessary operations, such as expansion, contraction or reflection of the simplex, are determined by reasonable comparisons of the objective function values; a simplex being the simplest volume in the $\mathrm{n}$-dimensional parameter space that consists of $n+1$ points. The biggest disadvantage of the Subplex method is the slow convergence to the end of the optimization, especially if the parameter combination is illconditioned, i.e. when strong discrepancies between the different parameters with respect to their influence on the objective function exist. Under such circumstances it is advisable to restart the algorithm. Another drawback of this method is the inability to abandon a local optimum in expectation for a global optimum.

\subsubsection{EGO}

The EGO algorithm [13] belongs to the group of surrogate methods. The general procedure consists in evaluating the true objective function at a number of arbitrary design points. Subsequently the method generates an approximation model of the objective function by means of the DACE predictor technique. In the next step the global optimum of the surrogate function and the uncertainty with respect to the true objective function is found by the aid of a genetic algorithm. The next design point for an evaluation of the true objective function is determined via the so called expected improvement function (EIF) which is created from the surrogate function and the uncertainty distribution. The use of the EIF provides for a balance between exploration of unknown places of the design space and exploitation of promising regions of the design space. Because of this dual character the method is very well suited to deal with multiple local optima. The only drawback is that the algorithm works sequentially after the generation of the first surrogate model leading to long turn-around times.

\subsection{Design variables}

The amount of evaluations during an optimization depends on the number of design variables. Because CFD computations are very time consuming, it is important to limit the number of design parameters. A trade off between the possibility of designing complex planforms and the number of design variables has to be made. Figure 2 shows the design variables: a) twist, b) sweep, c) taper, d) anhedral, e) starting point of transition to second profile (dimensions have been superelevated), f) starting point of blade tip area (Tipstart).

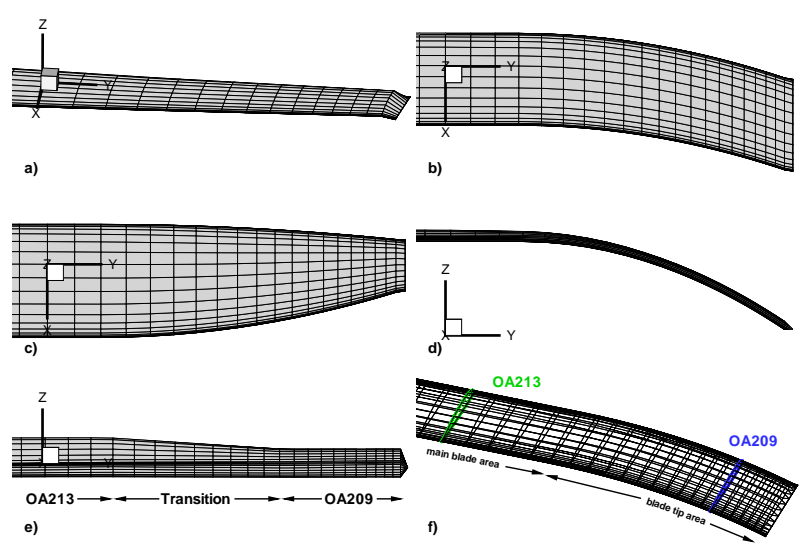

Figure 2: Design Parameters

Parameters a) to e) can be optimized separately or simultaneously. Changing the starting point of the blade tip will naturally only affect the design if at least one other parameter is chosen. The thickness of the blade can be controlled by varying the radial position of the transition between the two different airfoils. The twist is modified by changing the geometric twist over the blade span. While the geometric twist varies non-linearly over the blade span because of the two different profiles involved, it is ensured that the aerodynamic twist varies linearly. In order to avoid solidity effects the thrust weighted area is held constant according to equation 1). This means reducing the blade tip chord will result in an increased chord for the main part of the blade. Sweeping the blade is achieved by prescribing an inplane offset value for the quarter chord line at the outmost profile of the blade $(r / R=1.0)$. The sweep distribution is then given by a parabolic distribution law with zero deflection and zero slope at the starting point of the blade tip and the full deflection at the outmost section $(r / R=1.0)$. The anhedral of the blade is realized in the same manner.

1) $c_{e}=\frac{\int c * r^{2} d r}{\int r^{2} d r}$

For optimizations in hover the blade collective pitch angle $\theta_{0}$ is also added as a design variable. This way the rotor thrust is not fixed during the optimization. Considering two rectangular blades, the one with the higher collective pitch angle will always have the higher figure of merit as long as the flow is attached. Therefore the optimizer will strive towards high collective pitch angles assuring that the optimizer will reach the maximum figure of merit for each design configuration.

\subsection{Grid generation}

Once the blade surface has been constructed according to the new design variables the algebraic grid generator GEROS [10] is used for meshing the computational domain. All grids show a $\mathrm{C}-\mathrm{H}$ topology. The tab is modelled with a sharp trailing edge. The profiles at the root and tip cap are 
degenerated to a single line. Hover and forward flight optimizations are carried out on coarse meshes. In order to confirm the results the optimal rotor configuration at the end of each optimization run is being recomputed on the fine mesh. While $\mathrm{y}^{+}-$ values on the coarse meshes range between 3-4, for the fine meshes they lie below 1. Since GEROS is only capable of constructing monoblock meshes, grids have to be split afterwards in order to run the CFD computations in parallel.

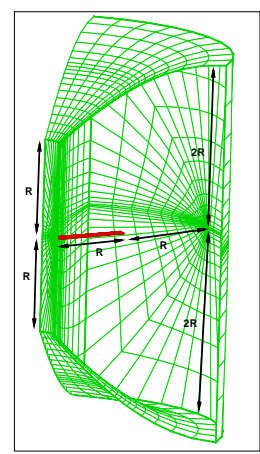

Figure 3: Hover

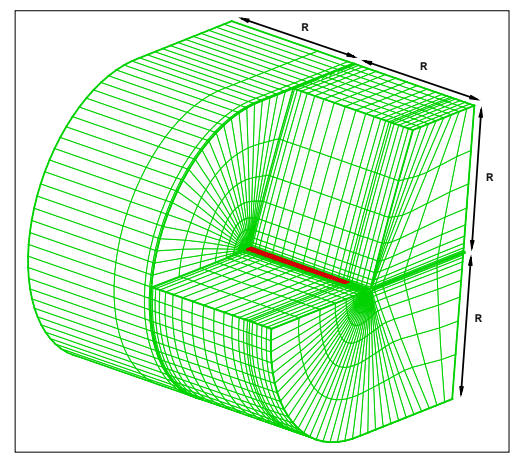

Figure 4: Single Blade Mesh (FF)

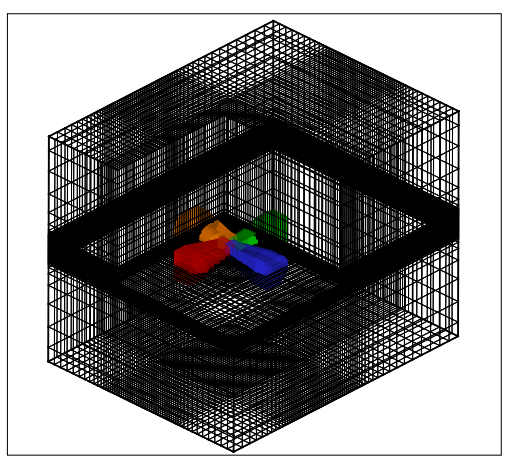

Figure 5: Chimera Configuration (FF)

\begin{tabular}{|c|c||c|c|}
\hline & Mesh & Number of elements c x r x n & Total number of elements \\
\hline \hline \multirow{3}{*}{ Hover } & Coarse & $60+28 \times 24+12 \times 8+24$ & 101367 \\
\cline { 2 - 4 } & Fine & $200+56 \times 60+24 \times 16+48$ & 1376256 \\
\hline \multirow{2}{*}{ FF } & Coarse & $96+32 \times 8+32+8 \times 24+16$ & 245760 \\
\cline { 2 - 4 } & Fine & $4 \times 192+64 \times 8+64+8 \times 48+32$ & $4 \times 1638400+3548160$ \\
\hline
\end{tabular}

Table 1: Number of elements for hover and FF grids: $c=$ number of elements in chordwise direction (number of elements on blade surface + number of elements for the wake), $r=$ number of elements in the radial direction (number of elements on the blade + number of elements from tip to farfield) and $n=$ number of elements in the normal direction (number of elements in the boundary layer + remaining number of elements to the farfield)

In hover the radial symmetry can be used to further reduce the computational domain as can be seen in Figure 3 . Therefore only $1 / 4$ of the domain has to be meshed. Table 1 summarizes the number of elements used for the coarse and the fine mesh. Optimizations in forward flight are undertaken with a single blade as depicted in Figure 4 thus also reducing the number of grid elements drastically. For confirmation optimization results are also recomputed using fine meshes (Figure 5) and the Chimera approach in order to include the interference between the rotor blades. The number of grid cells corresponds to the notation in hover while for the chimera configuration 3.5 million elements have to be added for the background grid.

\subsection{Aerodynamic module}

All CFD calculations in this paper have been achieved with the block-structured flow solver FLOWer from DLR [14]. The program solves the Reynolds-averaged Navier-Stokes equations, transformed into a moving blade-fixed coordinate system. The discretization of space and time is separated following the method of lines [12]. For the spatial discretization the cell-centered finite volume formulation is used. In order to avoid spurious oscillations, a blend of first and third order dissipative terms is introduced. Two layers of auxiliary points the so called ghost layers are added in order to assure second order accuracy across inner and outer block boundaries. In the present work a modified version of the 2-equation Wilcox k- $\omega$ model is applied.

Hover computations are accelerated with multigrid and local time stepping techniques. Forward flight computations are conducted with the dual-time stepping technique with a second order implicit time integration operator [11]. Elastic blade motions are accounted for by transfinite interpolation based on hermite polynomials. Free stream consistency for deforming grids is guaranteed by the geometrical conservation law [8].

\subsection{Structure module}

Blade dynamics and blade elasticity in this study have been calculated with the Comprehensive Rotorcraft code HOST [4] from Eurocopter. The program is mainly used for calculations of flight paths and stability issues and therefore disposes of a simple aerodynamic module based on liftingline theory. The aerodynamic coefficients are determined from 2D airfoil tables depending on 
the sectional Mach number and the angle of attack. Because of the high aspect ratio the rotor blade is modelled as a quasi-1D beam based on an extended Euler-Bernoulli formulation. For that purpose the blade is divided into small rigid segments which are connected via real or virtual joints. Moderate deformations in flap and lag direction as well as the elastic torsion are considered. On the other hand shear deformation and longitudinal expansion are neglected. Offsets between local center of gravity, bending and torsion are allowed in order to account for coupling effects between bending and torsion. The deformation of the blade is directly determined through the solution of the lagrange equation. The separation of space and time is achieved by a modal approach which at the same time helps reducing the number of degrees of freedom.

\subsection{Fluid-structure-coupling}

Evaluation of the rotor blade's aerodynamic performance is done using a weak coupling approach between the flow solver FLOWer and the simulation tool HOST. The calculations for each discipline are carried out alternately. The blade dynamics and deformations are passed once per revolution in the form of blade mode shapes and generalized coordinates. The aerodynamic loads resulting from integration of the surface pressure distribution (Figure 6) on the other hand are transferred in the rotating rotor hub system (Figure 7) as a function of radius and azimuth. Afterwards the loads are transformed into a fourier-series and transmitted to the CSDmesh via linear interpolation. Therefore the weak coupling procedure is very well suited for the analysis of periodic flight conditions [3]. Another advantage of this method is that it automatically provides a trimmed solution.

The goal of the method is to successively replace the aerodynamic loads computed by HOST's simple aerodynamic module with those obtained from the high-fidelity aerodynamic model. The correction of the aerodynamic loads changes the dynamic response of the rotor and vice versa until a steady periodic solution is reached. The procedure of the weak coupling is as follows:

1. Calculation of blade dynamics and elastic deformation for one revolution with HOST.

2. Evaluation of aerodynamic loads with FLOWer with respect to the previously computed blade motion and deformation (Figure 8).

3. The subsequent trim is performed with the corrected aerodynamic loads which read as follows:

2)

$$
\vec{F}^{n}=\vec{F}_{2 D}^{n}+\vec{F}_{3 D}^{n-1}-\vec{F}_{2 D}^{n-1}
$$

4. Steps (2) and (3) are repeated until the difference of the aerodynamic loads between two subsequent trim iterations tends to zero, namely:

3) $\Delta \vec{F}_{2 D}^{n}=\vec{F}_{2 D}^{n}-\vec{F}_{2 D}^{n-1} \rightarrow 0$

In this case the rotor is trimmed with the aerodynamic loads of the high-fidelity module.

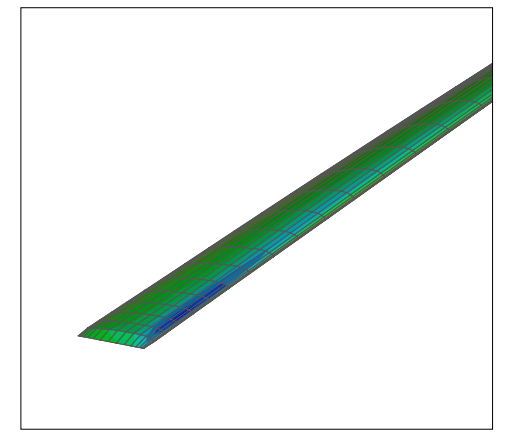

Figure 6: Surface pressure

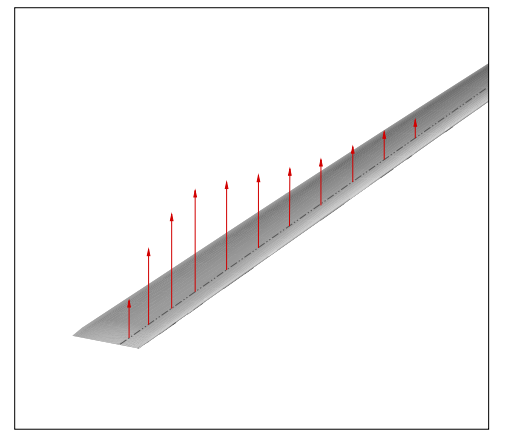

Figure 7: Forces on t/4-line

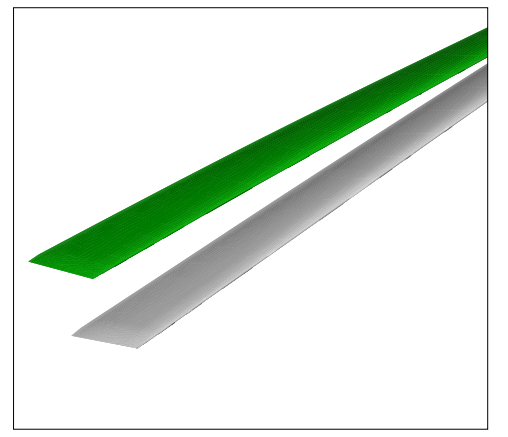

Figure 8: Mesh Motion

\subsection{Objective function}

A helicopter rotor requires the highest power in hover and fast forward flight. Therefore it is desirable to adapt the blade design such that the consumed power at those flight conditions becomes minimal or the attained thrust becomes maximal. One way to do so is to improve the rotor's efficiency which is represented by the figure of merit (FM). Although care has to be taken when comparing the figure of merit of different rotors (see section 2.2), it is chosen as the objective function for hover because optimizations can be carried out in an efficient manner.

When dealing with forward flight, power is not only consumed for the generation of thrust but also for propulsive force. Since no gauge as the FM exists for forward flight the objective function was taken to be the rotor torque coefficient $\mathrm{c}_{\mathrm{q}}$ which is directly proportional to the consumed power. In order to be able to compare different rotors, they have to be trimmed to match the same thrust and propulsive requirements. 
In the multi-objective case a weighted sum approach is favoured. In order to balance the different flight conditions correctly, the nominal values for $\mathrm{FM}$ and $\mathrm{C}_{\mathrm{q}}$ are divided by reference values resulting in equation 4 ):

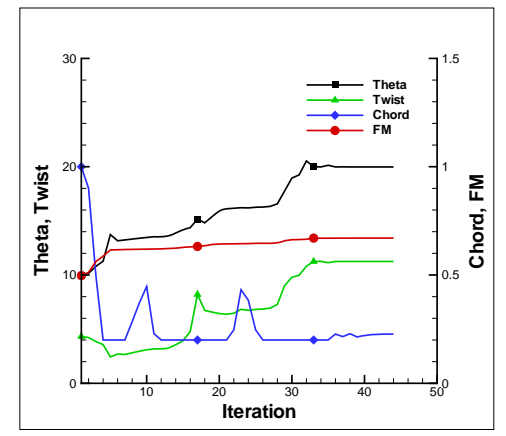

Figure 9: Congra

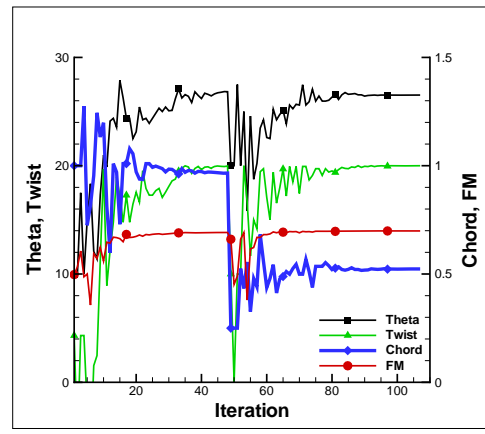

Figure 10: SubPlex

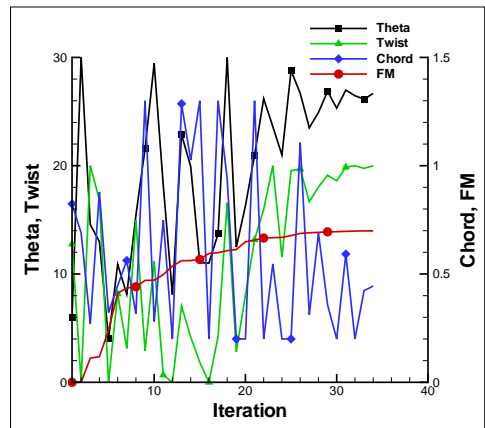

Figure 11: EGO

\begin{tabular}{|c||c|c|c|c|c|c|c|}
\hline \multirow{2}{*}{ Optimizer } & & & Congra & SubPlex1 & \multicolumn{2}{|c|}{ SubPlex2 } & EGO \\
\cline { 2 - 7 } Parameter & Start & Constraints & End & End & Start & End & End \\
\hline \hline Theta & 10.0 & 30.0 & 19.98 & 26.83 & 20.0 & 26.52 & 26.67 \\
\hline Twist & -4.32 & -20.0 & -11.36 & -19.98 & -10.0 & -19.99 & -20.0 \\
\hline Chord & 1.0 & 0.2 & 0.23 & 0.97 & 0.25 & 0.52 & 0.44 \\
\hline FM & 0.5073 & & 0.6712 & 0.6917 & 0.6604 & 0.6988 & 0.6993 \\
\hline
\end{tabular}

Table 2: Reference values for parameters and goal function of the optimization algorithms

\section{OPTIMIZATION RESULTS}

\subsection{Comparison of optimization algorithms}

Before starting the optimization studies for the challenging cases, i.e. fluid-structure coupled analyses with multiple parameters, the three different optimization algorithms presented in section 2.1 have been revised with respect to their efficiency. The test case was chosen to be a 3parameter - namely Theta, Twist and Chord optimization in hover with rigid blades. Table 2 shows the reference values for the parameters and the goal function for all three algorithms. The test case is very well suited for an efficiency assessment because the result is known a-priori from basic theory. The optimal hovering rotor as first described in [9] will offer a high twist and high taper, i.e. small blade tip chord (Table 2) resulting in a large blade root chord. Another challenging aspect for the optimizer is the different sensitivity of the chosen parameters. While theta and twist have a considerable influence on the goal function, the effect of the blade chord distribution plays a much less important role.

Figure 9 - Figure 11 show the convergence histories of the three optimizers for the three parameters and the goal function (twist values have been multiplied by -1 for illustration purpose). In the first case using the CONGRA algorithm it can be seen in Figure 9 that even with more than 40 iterations the optimizer does not reach the foreseen value for twist. Although the bound constraint for the tip chord is obtained, the optimization gives only a modest result for the FM attaining a value of 0.6712. The main improvements have to be attributed to the twist for which the constraint value is not reached. The reason for the bad performance of the algorithm can be found when looking at the step size for computing the gradient with finite differences. As a matter of fact no preliminary study in order to identify the correct step size has been done. Obviously the chosen step size was too large or too small resulting in a wrong gradient and therefore misleading the algorithm.

The SUBPLEX algorithm exhibits a much better behaviour. It closes the first optimization run after about 50 iterations with more promising values for theta and twist with a consequently higher value for FM of 0.6917 . Notice that the final value for theta is increased as a result of the higher twist value. While having found the optimal value for twist, SUBPLEX encounters difficulty when optimizing the tip chord. Although having changed the parameter throughout the optimization the 
optimizer returns to an almost rectangular blade at the end of the optimization. This is due to the much smaller influence of this parameter and also the known deficiency of the optimizer for premature convergence. Restarting the optimizer with a new parameter set close to the last best shows immediate remedy, i.e. a huge drop for the chord parameter can be observed in Figure 10 for iteration 49. The value for chord stays there while the value for twist quickly returns to the constraint value of -20.0 degrees. With a total of 107 iterations the algorithm attains a respectable result with a figure of merit of 0.6988 . Figure 11 demonstrates the calculation history for the EGO algorithm. The graph has been manipulated for illustrating reasons, i.e. the order of the calculations has been changed in favour to ascending FM-values. Nevertheless it can be deduced from the graph that the optimizer achieves a better value for the FM in a single run with fewer iterations than both the other algorithms. The performance of each of the algorithms will always depend on the initial and internal settings. Therefore a quantitative fair comparison will be hard to achieve. Qualitatively it can be stated that the EGO algorithm shows the most efficient procedure for this kind of engineering example and is least error-prone.

\subsection{Optimization in Hover}

At first the optimization framework is applied for hover flight conditions. The baseline configuration is the four-bladed ONERA 7A model rotor which was tested in the Modane wind tunnel [2]. The rotor has a rectangular planform, a radius of $2.1 \mathrm{~m}$ and a chord of $0.14 \mathrm{~m}$. The tip Mach number was chosen to be 0.646 . Three different parameter combinations will be presented in detail.

\subsubsection{Twist}

This simple test case is well suited to validate the functioning of the optimization framework, because a high twist is known to be beneficial in hover. In fact, by increasing the twist of the blade the lift production is shifted further inboard of the blade, see Figure 13. This reduces the strength of the tip vortex and gives a more uniform distribution of the induced velocities, therefore decreasing the induced power consumption. In Figure 12 the objective function (negative FM because optimization has been formulated as minimization problem) has been plotted as a function of theta and twist. It can be seen that the optimal parameter set is found for high theta and high twist values (deep blue). That is the region where the optimizer has chosen the most parameter sets (black squares). Figure 14 and Figure 15 show the polars of the baseline and optimized rotor on the coarse and on the fine grid respectively. The optimized rotor (red circles) has a higher FM over the whole range of thrust coefficients. Moreover the difference in FM of approximately 7 points between the baseline and optimized rotor on the coarse mesh is almost kept on the fine mesh ( 6 points). The best FM is also shifted towards higher thrust coefficients.

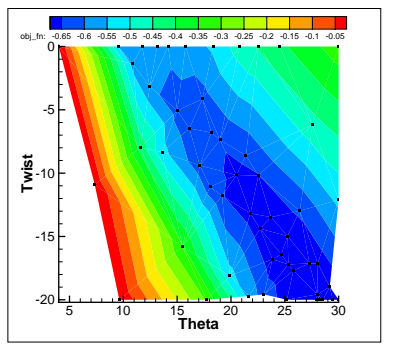

Figure 12: Function

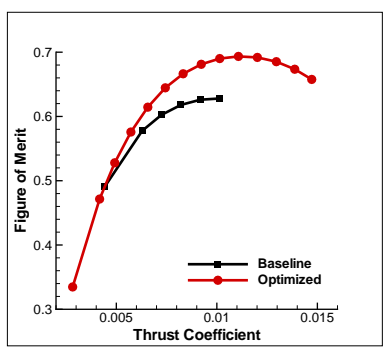

Figure 14: Polar coarse grid

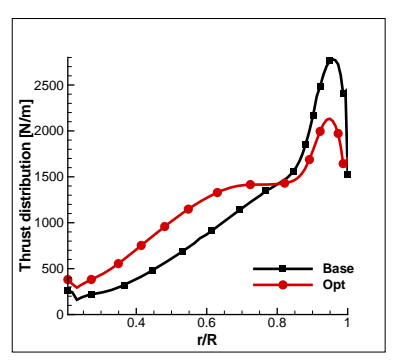

Figure 13: Thrust distribution

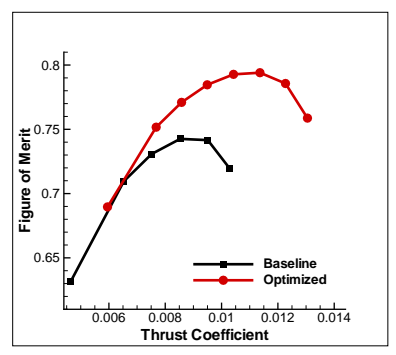

Figure 15: Polar on fine grid

\subsubsection{Twist and Sweep}

This optimization case is chosen in order to verify the functionality of the fluid-structure interaction. The second parameter (sweep) adds disproportionately more complexity to the design task since sweeping the blade tip induces a twisting moment which acts on the structure. This was not the case in the previous design problem. In Figure 17 the FM is plotted with respect to the design variables. The contour resembles an alpine topography with mountains representing high values of FM and valleys being related to low values of FM. Unlike in the previous case where the contour of the objective function exhibited a parabolic shape the objective function in this case is multimodal. Sweep is represented by a color coding, red values indicating a strong backward sweep, blue a strong forward sweep. Blue values can hardly be seen suggesting they are hidden in the valleys. In fact forward swept blades exhibit an intrinsically unstable behavior from a dynamic point of view. Most of the evaluations for strong forward swept blades have failed corresponding to low FM values and therefore cannot be seen in the plot. Contrary most of the peaks appear in green indicating that a moderate deflection of approximately $0.2^{*}$ chord seems to be the most beneficial. In fact the highest peaks can be found in the far right corner which signifies that the best 
parameter set is reached with high theta, high twist and a moderate sweep. At higher sweep values the elastic torsion becomes too big resulting in a degradation of the radial thrust distribution because the angle of attack of the outer sections has become too small. This can be seen in Figure 18 where the elastic torsion for three different cases (marked with a circle in Figure 17) is being compared. Clearly the elastic torsion depends on the amount of sweep, twist and collective pitch.

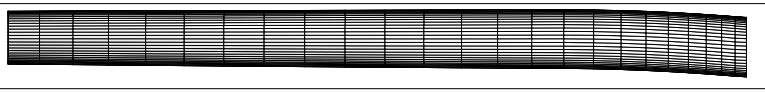

Figure 16:Top view of blade surface of optimal rotor

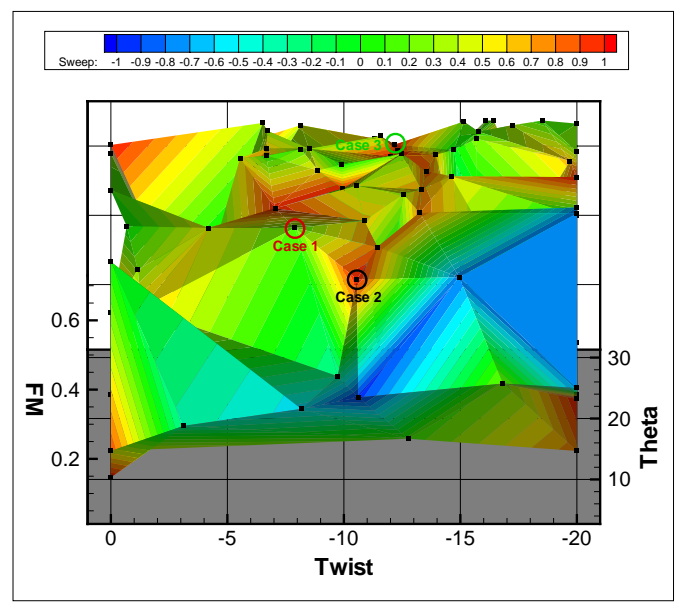

Figure 17: Objective Function

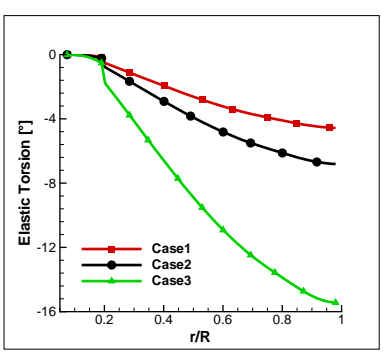

Figure 18: Elastic Torsion

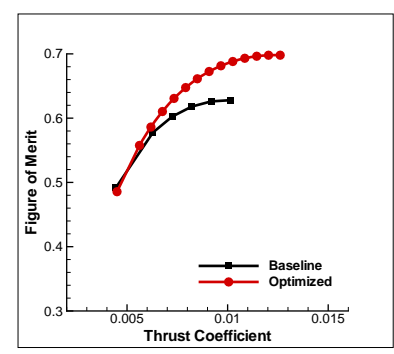

Figure 20: Polar on coarse grid

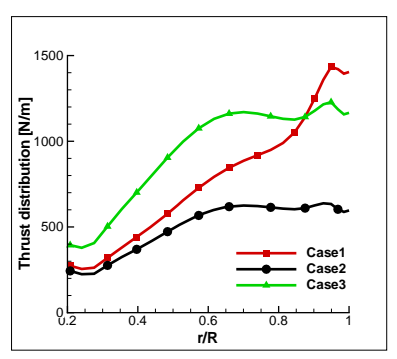

Figure 19: Thrust distribution

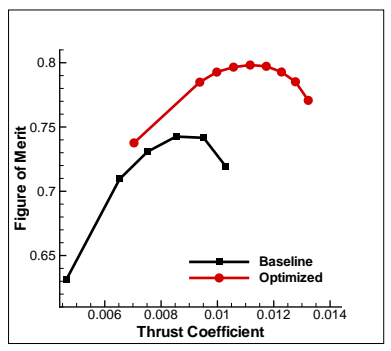

Figure 21: Polar on fine grid

\begin{tabular}{|c||c|c|c|c|}
\hline & Theta & Twist & Sweep & Tor_el \\
\hline \hline Case1 & 18.3 & -7.9 & 0.17 & -4.6 \\
\hline Case2 & 17.0 & -10.6 & 1.0 & -6.8 \\
\hline Case3 & 29.0 & -12.2 & 1.0 & -15.4 \\
\hline Opt & 30.0 & -16.4 & 0.15 & -6.0 \\
\hline
\end{tabular}

Table 3: Design parameters and elastic torsion for three different cases

Summing up the values for theta, twist and elastic torsion (Tor_el) in Table 3 for the different cases shows that the angle of attack of the outmost station is the biggest for case 1 with $5.8^{\circ}$. Case 3 shows an angle of $1.4^{\circ}$ and for case 2 the angle is even slightly negative. As can be observed in Figure 19 this results in a significant decrease of thrust in the outer section of the blade for case 2 and case 3 compared to case 1 which exhibits an almost triangular thrust distribution.

Figure 20 illustrates the polar of the baseline and the optimal parameter set (see also Table 3 ) on the coarse grid. A considerable improvement of about 7 points can be recorded. Again this benefit can be maintained on the fine grid (Figure 21) and is also being shifted to higher thrust coefficients.

\subsubsection{All Parameters}

Adding parameters means increasing the optimizer's choice but also the complexity of the problem. The present analysis needed more than 200 evaluations in order to converge to an optimal parameter set. Furthermore combining certain parameters may create interdependencies. Figure 22 depicts the correlation between the different parameters and the FM. It can be seen that when the objective function is confined to a scale of 0.69 to 0.71 clear relations for each individual parameter exist. That means that for an objective function in this interval theta has to be in a range of $25^{\circ}$ to $30^{\circ}$ and the twist will be between -10 and $-20^{\circ}$. This is not surprising since it is in accordance with the other optimization runs. The same is true for the tip chord which lies between 0.5 and 0.8 which helps to unload the tip decreasing the intensity of the tip vortex and improving the radial thrust distribution. Unlike in section 3.2.2 where a moderate sweep was the best choice in this optimization an almost linear trend can be observed towards higher sweep values nearly reaching the constraint of 1.0. The reason for this can be found when correlating sweep with chord as is achieved in the upper right graph in Figure 22 with a color map. Then it becomes obvious that the higher sweep values always match with a small tip chord resulting in a smaller twisting moment and consequently lesser elastic torsion. The result for anhedral seems surprising at first since the optimal value is very 
close to zero. Taking a closer look at the geometry in Figure 23 reveals that an anhedral can be seen though. The reason for this lies in the combination of twist and sweep which induce an anhedral when applied in the correct order. Because the blade is twisted at first and swept afterwards, the blade tip is not only deflected inplane but also out of plane. The reader should note that the starting point of the tip also influences the sweep and anhedral angle while nominal values stay the same. Since the optimal rotor shows a considerable twist and sweep, a distinct anhedral angle of about $32^{\circ}$ is reached. This might also be the reason why a small tip region is preferred by the optimizer. As shown in the previous section a small tip region in combination with a small tip chord helps unloading the tip and the combination with a strong sweep generates a desirable nose down elastic twist. The scope for the profile transition (from OA213 to OA209) is shifted further inboard between 0.5 and 0.7 . This is quite reasonable since the optimal rotor operates at a considerably high collective pitch angle (even when taking into account the twist) and a thinner profile helps reducing the local Mach number and therefore the shock on the profile.
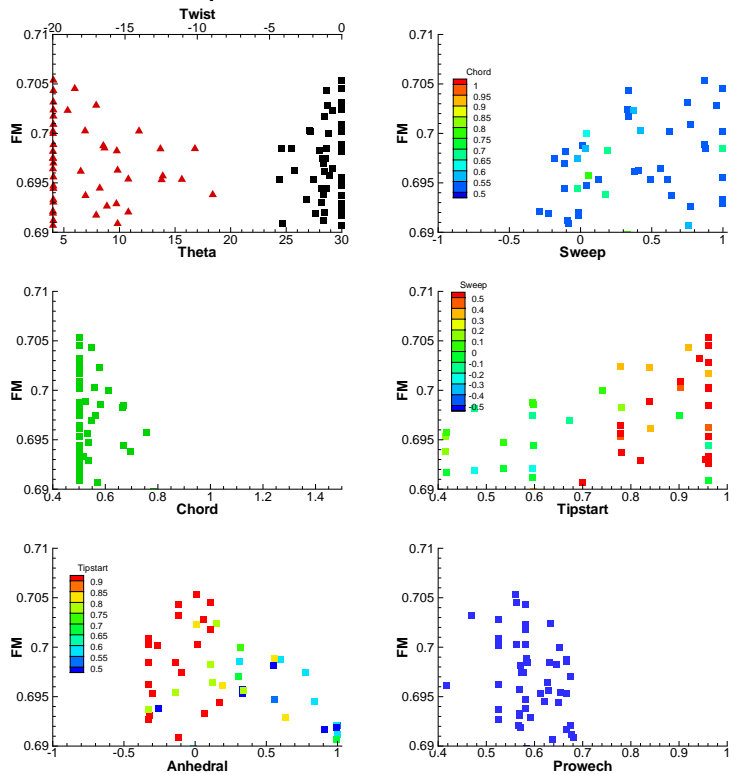

Figure 22: Correlation between parameters and FM

Topview

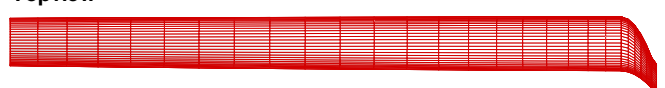

Sideview

Figure 23: Blade surface of optimal rotor (w/o twist)

Figure 23 shows the optimal hovering rotor for an optimization with all parameters. The tip chord and the sweep can be viewed in the top view. The parameter values are summarized in Table 4.

\begin{tabular}{|c|c|c|c|}
\hline Theta & Twist & Chord & Anhedral \\
\hline 30.0 & -20.0 & 0.50 & 0.008 \\
\hline \hline Sweep & Tipstart & Protrans & \\
\hline 0.87 & 0.955 & 0.557 & \\
\hline
\end{tabular}

Table 4: Parameter set of the optimal rotor

\subsection{Optimization in Forward flight}

Optimizations in forward flight are somewhat more cumbersome than in hover since unsteady CFD computations have to be conducted. The flight condition is a fast forward speed $(\mu=0.4)$ case with a tip Mach number of 0.646 . The rotors were trimmed to a total thrust of $4400 \mathrm{~N}$ and a propulsive force of $563 \mathrm{~N}$.

In Figure 24 the objective function (torque coefficient) is presented as a function of the twist rate. The squares correspond to the evaluations during the optimization (single blade computations), the circles represent the results from the full chimera computations. As can be seen a close correlation between the single blade and the full chimera evaluations exists. This can be attributed to the fact that the blade vortex interaction for this flight condition is very limited. Although differences become apparent when detailed information such as normal force over azimuth is compared, the effects become less influential when considering integral values.

Regardless of the computation technique (single blade or chimera) it can be deduced from Figure 24 that the optimal twist for forward flight is about $-6^{\circ}$ which is not very far from the twist rate of the reference rotor $\left(-4.32^{\circ}\right)$. Changing the twist rate to smaller or higher values leads to a worsening of the objective function. Although in general the idea for optimizing a blade in forward flight is the same than in hover, i.e. shifting the thrust distribution inboard as far as possible, the realization is far more difficult because of the conflicting requirements in the different azimuthal quadrants.

Nevertheless when analysing the lower end of the twist spectrum, e.g. a slightly twisted blade $\left(-3.1^{\circ}\right)$ with the optimal twisted blade $\left(-5.96^{\circ}\right)$ that is exactly the case. Regarding Figure 25 and Figure 26 it can be seen that the slightly twisted rotor produces more thrust on the outer part between 0 and $210^{\circ}$ azimuth. This is also illustrated for an azimuth position of $120^{\circ}$ in Figure 27. In consequence the slightly twisted rotor consumes more power in the outer region of the rotor which is demonstrated by the red color in Figure 28, thus resulting in a small power degradation of 0.4 kW. 


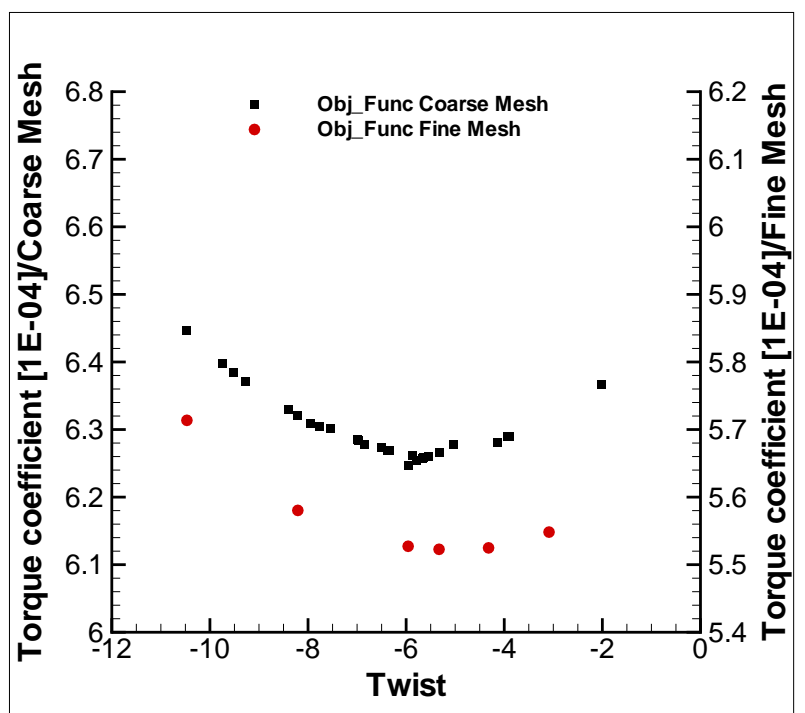

Figure 24: Objective function on coarse and fine mesh

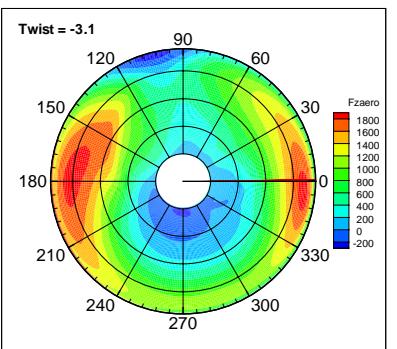

Figure 25: Thrust distribution for slightly twisted blade

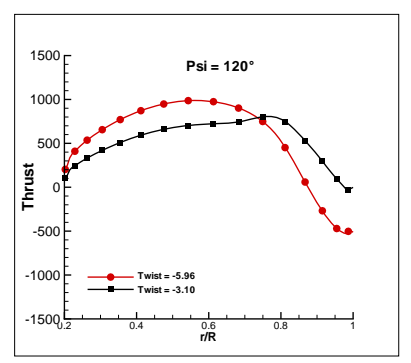

Figure 27: Radial thrust distribution at $p s i=120^{\circ}$ for slight and optimal twisted blade

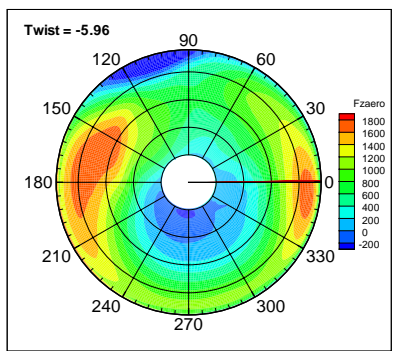

Figure 26: Thrust distribution for optimal twisted blade

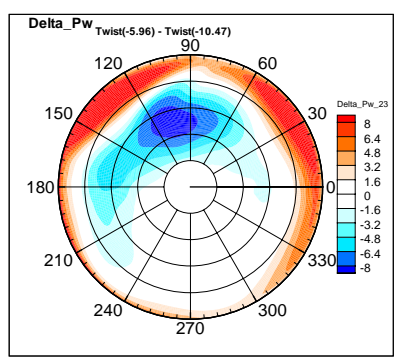

Figure 28: Power difference between slight and optimal twisted blade

As stated before this trend cannot be continued to very high twist rates. Figure 29 and Figure 30 compare the thrust distribution of a highly twisted blade $\left(-10.47^{\circ}\right)$ and the optimally twisted blade. As in the previous example the rotor with the higher twist rate generates more thrust at the inboard sections of the blade which is shown for an azimuth position of $120^{\circ}$ in Figure 31 . However, the thrust distribution for the highly twisted rotor cannot be regarded as beneficial anymore because a huge negative thrust region has developed on the outer part of the blade between 90 and $150^{\circ}$ azimuth. This cannot be prevented, since the trim objectives have to be obtained. In order to compensate for the strong downward thrust of the outboard sections the blade has to be adjusted to a high collective pitch resulting in a power penalty in this region. Adding up the different contributions yields an overall consumed power of $104.4 \mathrm{~kW}$ for the highly twisted blade and $101.0 \mathrm{~kW}$ for the optimally twisted blade leaving a benefit of $3.4 \mathrm{~kW}$.

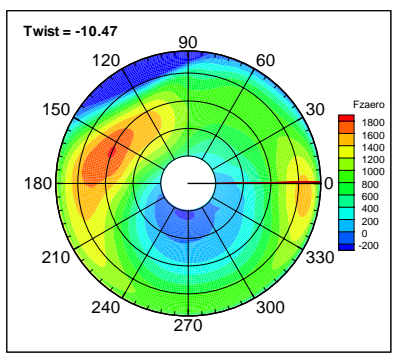

Figure 29: Thrust distribution for highly twisted rotor

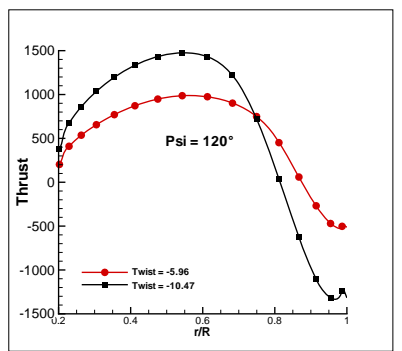

Figure 31: Radial thrust distribution at $\mathrm{psi}=120^{\circ}$ for high and optimal twisted rotor

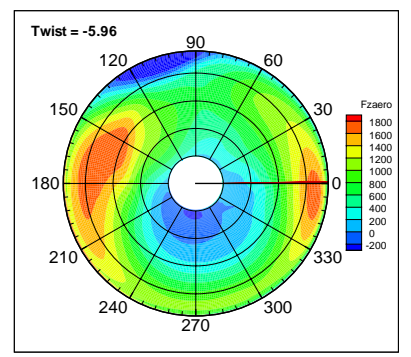

Figure 30: Thrust distribution for optimal twisted rotor

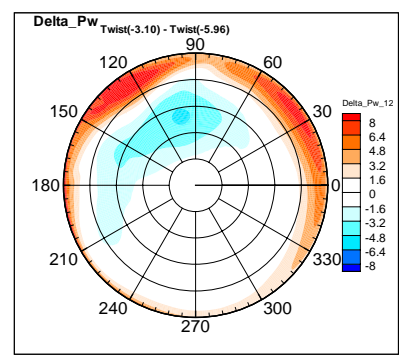

Figure 32: Power difference between high and optimal twisted rotor

\subsection{Multi-objective Optimization}

Optimizing a helicopter rotor for a single flight condition is a necessary task in order to understand the effect of different design parameters. On the other hand it is not a relevant case for real rotors since a rotor optimized for hover will suffer from huge performance deficits in forward flight and vice versa. Thus it is of great interest to design a rotor for hover and forward flight simultaneously. Since the optimization in forward flight has already shown to be time consuming the simplest case of twist optimization has been chosen for the multi-objective case. The objective functions and flight conditions are chosen as in the single-objective case which means that the collective pitch has to be added as a design parameter. For the combination of the two flight conditions a weighing of function approach (WOF) is chosen according to equation 4).

Five different sets were computed: pure hover $\left(w_{1}\right.$ $\left.=0.0, w_{2}=1.0\right)$, hover predominance $\left(w_{1}=0.25\right.$, $\left.\mathrm{w}_{2}=0.75\right)$, equal weighing $\left(\mathrm{w}_{1}=0.5, \mathrm{w}_{2}=0.5\right)$, forward flight predominance $\left(w_{1}=0.75, w_{2}=\right.$ 
0.25), pure forward flight $\left(w_{1}=1.0, w_{2}=0.0\right)$. Before the two objectives can be summed up they are divided by reference values as can be seen in equation 4) in order to reach unity in both cases. Otherwise the chosen weights would be meaningless in terms of an equal comparison. Figure 33 shows the result of the different WOF sets. For the pure hover set the result as in the single objective case e.g. maximum twist $\left(-20^{\circ}\right)$ is reached. Therefore the hover objective function (Obj_hov) is equal to one meaning the FM has attained the reference value. For the pure forward flight set the reference value is also reached but at a slightly smaller twist rate as in the single objective case. When closely observed this is not a disturbing issue since it can be seen in Figure 24 that the gradient of the objective function in this region is quite small. In fact, when comparing the different computations within this set it becomes clear that an increase in twist rate of about $1^{\circ}$ results in only a petite performance degradation. Furthermore the convergence in the MDO case is slower than in the SDO because optimizations are done with two parameters (collective pitch angle). Therefore a better performance at a higher twist rate could have also been reached if the optimization had been carried out further.

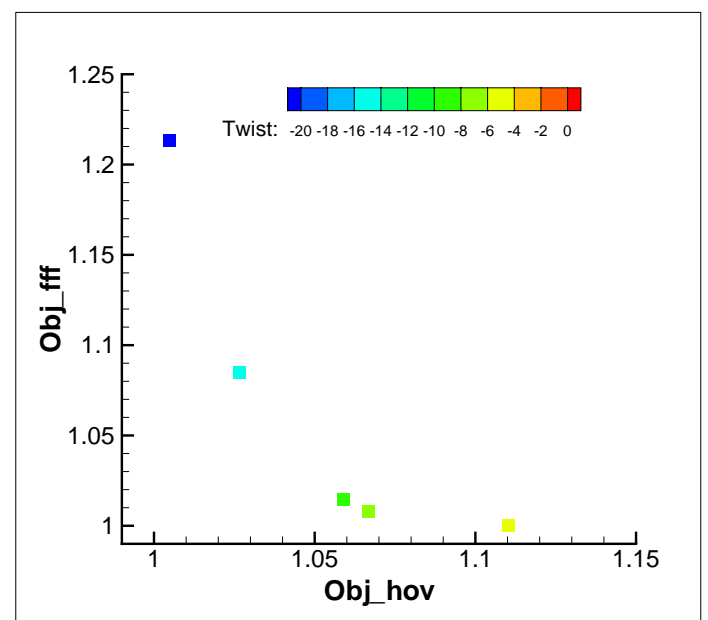

Figure 33: Multidisciplinary optimization of twist with WOF approach

Increasing the twist rate to values of about $-9^{\circ}$ (green squares) shows a good improvement for the hover objective function without huge performance degradation in forward flight as can be seen in Figure 33. In this case the two objectives are weighed equally. Shifting the importance further to the hover case $\left(w_{1}=0.25\right.$, $w_{2}=0.75$ ) pushes the twist rate to values of about $-14^{\circ}$ and results in a strong performance penalty for forward flight.

\section{CONCLUSION}

An optimization framework for helicopter rotors in hover and forward flight based on high-fidelity coupled CFD/CSM analyses has been presented. At first the mesh generation process and the fluid structure module have been illustrated. In a second step the effectiveness of different optimization algorithms has been revised showing the superiority of the EGO algorithm over the traditional gradient and pattern search method. Optimizations in hover reveal the importance of the twist distribution for this flight condition. In comparison with other parameters the principal gains result from an increased twist rate. Although a non-linear twist distribution would be favourable the main improvements are achieved with a linear twist giving the advantage of having only one design parameter.

Optimizations including the sweep as design parameter demonstrate the necessity of taking into account the fluid structure interaction. While forward swept blades exhibit an unstable behaviour moderate backward swept blades show a favourable influence on the elastic torsion which improves the rotor's efficiency. Furthermore the test case showed the unconditional need for a global optimization algorithm which does not get stuck in a local optimum.

In hover the variation of the chord can add only a slight additional improvement. Nevertheless it is important in combination with other parameters. Furthermore this parameter will gain more importance for forward flight optimizations since sweeping the blade without tapering will induce huge twisting moments quickly reaching the structural limits of the blade.

An optimization of a hovering rotor considering many design parameters increases the parameter space and therefore the possibility of reaching a higher FM. On the other hand it has become clear that more parameters increase the complexity of the design problem and therefore the chance of missing the global optimum since the objective function has become highly multimodal. Another important aspect is that the parameters heavily interact with each other making it difficult to isolate individual effects. The major blade changes have been made to the blade tip, since this is the most effective part of the blade. It will be left to further researches to investigate the possibility of stronger modifications to the inner part of the blade. The starting point should be a parameterization for individual blade sections which will in turn increase the number of design variables.

In forward flight it could be demonstrated that the chosen procedure of optimizing with only a single blade is valid. The trend of the objective function was very well predicted showing an almost constant offset between the single blade and the full chimera approach. As expected a high twist rate in forward flight increases the torque coefficient dramatically. Moderate twist rates do 
not degrade forward flight performance too much but offer substantial improvements for the hover condition.

This could also be shown in the MDO case. Increasing the twist rate to values of up to $-9^{\circ}$ gives reasonable improvements in hover while not strongly degrading forward flight performance. Since a lot of computations have to be done in MDO only few parameters can be analysed. Strategies for lowering computational costs seem to be a key element for future researches.

In summary it can be said that very promising results have been achieved. For the first time CFD computations have been extensively used inside an optimization. The superiority of this approach for complex geometries in comparison with traditional methods could be demonstrated. Although the calculations are quite time consuming different strategies have been adopted to make such optimizations feasible in the scientific context. Nevertheless some major drawbacks still exit, i.e. the properties of the structure are fixed during an optimization. Although it is expected that the structural properties can be adjusted to meet the predicted aerodynamic expectations it cannot be guaranteed. Furthermore it cannot be stated if the optimization had proceeded in the same way if the structural properties had been changed during the optimization. Another interesting aspect is the applicability of the outlined procedure for other flight regimes. More challenging flight conditions such as BVI-cases will need additional modeling techniques on the CFD side. Last but not least methods for reducing the computational costs will be crucial for an industrial acceptance.

\section{REFERENCES}

[1] B.M. Adams. The dakota toolkit for parallel optimization and uncertainty analysis. In SIAM Conference on Optimization, Boston, MA, May 2008.

[2] M. Allongue, J.P. Drevet. New rotor test rig in the large Modane wind tunnel. In $15^{\text {th }}$ European Rotorcraft Forum, Amsterdam, Netherlands, 1989.

[3] A.R.M. Altmikus, S. Wagner, P. Beaumier, G. Servera, A comparison: Weak vs. strong modular coupling for trimmed aeroelastic rotor simulations. In American Helicopter Society $58^{\text {th }}$ Annual Forum, Montreal, Canada, 2002.

[4] Bernard Benoit, André-Michel Dequin, Konstantin Kampa, Wolfgang Von Grünhagen, Pierre-Marie Basset, and Bernard Gimonet. Host, a general helicopter simulation tool for germany and france. In American Helicopter Society $56^{\text {th }}$ Annual Forum, 2000.

[5] A. Chattopadhyay and Y. Danny Chiu. An enhanced integrated aerodynamic load/dynamic optimization procedure for helicopter rotor blades. Contractor Report NASA CR-4326, National Aeronautics and Space Administration, Langley Research Center Hampton, VA 23665-5225, Oct 1990.

[6] K. Collins, J. Bain, N. Rajmohan, L. Sankar, T. A. Egolf, R. D. Janakiram, K. Brentner, and L. Lopes. Toward a high-fidelity helicopter rotor redesign framework. In American Helicopter Society $64^{\text {th }}$ Annual Forum, 2008.

[7] A. Dumont, A. LePape, J. Peter, and S. Huberson. Aerodynamic shape optimization of hovering rotors using a discrete adjoint of the rans equations. In American Helicopter Society $65^{\text {th }}$ Annual Forum, 2009.

[8] A.L. Gaitonde and S. Fiddes. A threedimensional moving mesh method for the calculation of unsteady transonic flows. Technical Report 483, University of Bristol, Sep 1993.

[9] A. Gessow and G.C.Jr. Myers. Aerodynamics of the Helicopter. Frederick Ungar Publishing Co., 7. Edition, 1983.

[10] M. H. L. Hounjet, C.B. Allen, L. Vigevano, N. Trivellato, A. Pagano, A. D'Alascio, and N. Jobard. Outline and application of geros: a european grid generator for rotorcraft simulation methods. Technical Report, NLR, 1998.

[11] A. Jameson. Time dependent calculations using multigrid, with applications to unsteady flows past airfoils and wings. In AIAA Paper, number 91-1596, 1991.

[12] A. Jameson, W. Schmidt, and E. Turkel. Numerical solutions of the euler equations by finite volume methods using runge-kutta time-stepping schemes. In AIAA Paper, volume 81, 1981.

[13] Donald R. Jones, Matthias Schonlau, and William J. Welch. Efficient global optimization of expensive black-box functions. Journal of Global Optimization, 13:455-492, June 1998.

[14] J. Raddatz and J. Fassbender. Block structured Navier-Stokes solver FLOWer. MEGAFLOW - Numerical Flow Simulation for Aircraft Design. Vol.89 of Notes on Numerical Fluid Mechanics and Multidisciplinary Design, 27-44, Springer, 2005.

[15] J. G. Leishman. Principles of helicopter aerodynamics. Cambridge University Press, $2^{\text {nd }}$ Edition, New York, 2006.

[16] A. LePape. Numerical aerodynamic optimization of helicopter rotors: Multiobjective optimization in hover and forward flight conditions. In $31^{\text {st }}$ European Rotorcraft Forum, September 2005. 
[17] A. LePape and P. Beaumier. Numerical optimization of helicopter rotor aerodynamic performance in hover. In $31^{\text {st }}$ European Rotorcraft Forum, September 2005.

[18] Joanne L. Walsh, Katherine C. Young, Frank J. Tarzanin, Joel E. Hirsh, and Darrell $\mathrm{K}$. Young. Optimization issues with complex rotorcraft comprehensive analysis. In $7^{\text {th }}$ AIAA/USAF/NASA/ISSMO Symposium on Multidisciplinary Analysis and Optimization, number AIAA 98-4889, September 1998.

[19] K.A. Yuan and P.P. Friedmann. Aeroelasticity and structural optimization of composite helicopter rotor blades with swept tips. Contractor Report 4665, National Aeronautics and Space Administration, Langley Research Center, Hampton, Virginia, May 1995. 